\title{
Trimeric GITRL-Fc OMP-336B11
}

National Cancer Institute

\section{Source}

National Cancer Institute. Trimeric GITRL-FC OMP-336B11. NCI Thesaurus. Code C143155.

A Fc-eng ineered human fusion protein composed of two trimers of tumor necrosis factor (ligand) superfamily, member 18 (TNFSF18; GIT RL) linked to an immunog lobulin Fc domain (GIT RL-Fc), with potential immunostimulatory and antineoplastic activities. Upon administration, trimeric GIT RL-Fc OMP-336B11 targets, binds to and activates its costimulatory surface receptor (glucocorticoid-induced tumor necrosis factor receptor (GITR; TNFRSF18) expressed on T-lymphocytes and certain tumor cell types. This activates T-lymphocytes, causes T-lymphocyte proliferation and suppresses the activity of regulatory T-cells (T reg). This promotes cytotoxic T-lymphocyte (CT L)-mediated killing of tumor cells. GITRL, a member of the tumor necrosis factor (TNF) family of ligands, functions to activate the co-stimulatory receptor GITR to enhance T-cell modulated immune responses. 